\title{
Le Cours de linguistique générale et l'histoire du saussurisme
}

\author{
Anne-Gaëlle Toutain \\ Université de Berne, Suisse \\ annegaelletoutain@yahoo.fr
}

\section{Résumé}

Il apparaît que l'histoire du saussurisme tout entière, depuis la première publication des idées de Saussure sur la linguistique générale jusqu'au récent et contemporain néo-saussurisme, est marquée par la même lecture idiomologique de Saussure. Le Cours de linguistique générale, bien que critiquable, apparaît ainsi comme un maillon -le premier-d'une histoire qui le dépasse: celle du recouvrement de la rupture saussurienne, et il est à cet égard très remarquable qu'il soit finalement le moins opacifiant de tous. Un siècle après le Cours de linguistique générale, on ne peut donc qu'appeler à un «retour à Saussure», à partir de tous les textes disponibles et du travail philologique auquel s'appliquent de nombreux saussuriens, et qui substitue la réflexion épistémologique à la vénération de l'autographe et à la problématique de l'histoire des idées, dans laquelle le terme d'histoire conserve un sens regrettablement empirique. Que nous enseigne, sur la langue et sur la linguistique, ce singulier destin de l'œuvre saussurienne de linguistique générale?

Mots clés : Cours de linguistique général [CLG], Saussure, lecture philologique, corpus saussurien

\section{The Course of General Linguistics and the History of Saussurism}

\begin{abstract}
It appears that the whole history of Saussurism, from the first publication of Saussure's ideas on general linguistics to the recent and contemporary neo-saussurism, is marked by the same idiomological reading of Saussure. The Course of General Linguistics, although open to criticism, appears as a link -the first- of a history that surpasses it: a history of the recovery of the Saussurean rupture, and it is in this respect very remarkable that it is ultimately the least unclear of all. A century after the Course of General Linguistics, one can only call for a "return to Saussure", on the basis of all available texts and the philological work to which many Saussurians apply, and which substitutes the epistemological reflection for the veneration of the autograph and the problematic of the history of ideas, in which the term history retains a regrettably empirical meaning. What does this singular fate of the Saussurean work of general linguistics teach us about language and linguistics?
\end{abstract}


Nous fêtons cette année les cent ans du Cours de linguistique générale, livre à bien des égards inaugural pour la linguistique du XXe siècle, et l'on peut, à cette occasion, s'interroger sur le statut de ce dernier, à un siècle de distance et alors que la publication, à partir des années 1950, de ses «sources manuscrites ${ }^{1} »$, en a remis en cause la légitimité. Ce livre a pendant quelque quarante ans constitué l'unique référence des études saussuriennes, et pour ces premiers lecteurs de Saussure, les sources manuscrites, notes d'étudiants ou notes autographes, sont ainsi nécessairement venues en complément du texte rédigé par Bally et Sechehaye. Cette position demeure celle de lecteurs contemporains de Saussure, tels, par exemple, Claudine Normand, qui, dans son ouvrage sur Saussure paru en 2000, affirmait, à propos du Cours de linguistique générale: «[...] ce texte, maintes fois réédité et traduit, je continuerai selon la tradition à l'appeler Saussure. Il ne s'agit évidemment pas d'ignorer les travaux philologiques mais de les laisser à leur rôle de complément et correction éventuelle, en refusant qu'ils fassent écran à une première réflexion sur le Cours en tant que texte, seul texte aisément lisible de façon suivie, à lire comme tant d'autres l'ont fait pour leur plus grand profit» (Normand, 2000: p. 12), ou encore Jürgen Trabant, qui conclut son article «Faut-il défendre Saussure contre ses amateurs? Notes item sur l'étymologie saussurienne» (2005) en affirmant: "Le Saussure "authentique" est tragiquement condamné à rester le bouffon du Cours». (Trabant, 2005: p. 124). D'autres, en revanche, considèrent le Cours de linguistique générale comme un texte «apocryphe», tels Simon Bouquet ${ }^{2}$, Rossitza Kyheng ${ }^{3}$ ou François Rastier qui, tout récemment encore, dans Saussure au futur (2015), affirmait: «Ainsi, l'accès à la pensée de Saussure a tout à la fois été permis et entravé par le Cours, qui a tous les caractères d'une vulgate: indispensable, partout cité, sans valeur scientifique. Non seulement il fait l'impasse sur les développements épistémologiques par lesquels commençait le deuxième cours à l'Université de Genève, mais surtout il minimise l'apport de Saussure à la linguistique de la parole. En somme, outre les rares études publiées par Saussure, les manuscrits sont les seuls écrits authentiques à partir desquels l'on puisse travailler, les cahiers d'étudiants et le Cours n'étant que des documents annexes et complémentaires». (Rastier, 2015: p. 17-18), allant ainsi pour sa part jusqu'à exclure du corpus saussurien les notes des étudiants ${ }^{4}$. Il existe bien entendu

1 Je me réfère ici bien entendu au titre de l'ouvrage de Godel paru en 1957. Voir Godel (1957).

2 Qui affirmait ainsi par exemple en 2008, dans «Ontologie et épistémologie de la linguistique dans les textes originaux de Ferdinand de Saussure»: «La pensée saussurienne, historiquement reçue du Cours de linguistique générale et reconnue comme ayant suscité un renouveau dans les sciences du langage, est désormais à reconsidérer et à réévaluer sur la base de l'ensemble des textes originaux connus à ce jour. Et il n'est pas exagéré de parler à cet égard, au point de vue de la réception, d'un "second Saussure", comme on parle d'un "second Wittgenstein" - à cette différence près que le "second Saussure" est chronologiquement antérieur au "premier", et que le "premier" doit être considéré comme un "Pseudo-Saussure"». (Bouquet, 2008: p. 1), puis: «[...] la pensée saussurienne fait régulièrement l'objet de malentendus méconnaissant la leçon des textes originaux - le Cours apocryphe et posthume de 1916 étant encore souvent brandi comme un ouvrage dont Ferdinand de Saussure serait l'auteur». (Bouquet, 2008: p. 2). Voir également infra, la section 4.

3 Qui, dans «Principes méthodologiques de constitution et d'exploitation du corpus saussurien» (2007), propose une «structuration interne du corpus saussurien selon les degrés d'authenticité des textes» (Kyheng, 2007: p. 2) et exclut le Cours de linguistique générale de ce corpus: «Résumons : le Cours de linguistique générale - qui, pendant des décennies et pour les raisons qu'on connaitt, a revendiqué l'autorité auctoriale de Ferdinand de Saussure en jouant le rôle de texte unique pour le large public, et de texte principal pour les spécialistes - est un texte pseudo-authentique à l'égard de Saussure, et ne peut figurer dans le corpus saussurien à aucun titre». (Kyheng, 2007: p. 17).

4 Que Kyheng, pour sa part, qualifie en revanche de textes «quasi-authentiques», et qu'elle inclut dans le corpus saussurien, tout en précisant: «La séparation entre textes authentiques et textes quasi-authentiques est la frontière indispensable entre ce que dit/écrit Saussure, et ce que les interlocuteurs font dire à Saussure. La fiabilité des textes quasi-authentiques ne peut jamais égaler celle des textes authentiques de Saussure du premier volet, et là où la comparaison est possible, le texte autographe doit être privilégié». (Kyheng, 2007: p. 10). 
tous les intermédiaires possibles entre ces deux positions, d'ailleurs plus ou moins radicales, comme en témoigne, par exemple, la modération de Claudine Normand, qui pense possible une "coexistence entre les travaux philologiques (disons la "saussurologie") et un travail de diffusion et réflexion renouvelée sur Saussure» (Normand, 2000: p. 16) , $^{5}$ et qui a elle-même travaillé sur les manuscrits de Saussure, mais il me semble pour ma part que ces deux problématiques sont, à certains égards, trop simples. À l'encontre de la seconde, il importe de souligner que s'il paraît de bonne méthode de privilégier les propos de Saussure les moins médiatisés (bien que les notes autographes, demeurées à l'état de brouillon, ne soient pas sans poser problème), il ne suffit pas -et, comme nous le verrons, les travaux des tenants du «néo-saussurisme» en témoignent de manière exemplaire- de disposer $\mathrm{du}$ «vrai texte» pour le comprendre, et il faut rappeler, à cet égard, après d'autres, qu'il y a longtemps que les notes d'étudiants et de nombreux textes autographes de Saussure sont disponibles, corpus que les manuscrits découverts en 1996 viennent compléter, fût-ce de manière décisive, plutôt qu'ils n'en bouleversent la représentation. Par ailleurs, la notion même d'authenticité est à interroger: comme le souligne Claudine Normand $^{6}$, «[o]n peut juger de la portée d'un texte par ce qu'il apporte lui-même et non par référence à un auteur solitaire et sacralisé» (Normand, 2000: p. 13), et c'est même ce qui importe avant tout, au risque, sinon, de substituer la caution d'un auteur à la réflexion et à l'analyse épistémologiques ${ }^{7}$. La première problématique me paraît pour sa part trop naïvement historique: l'histoire des textes et de leur réception, l'histoire «sociologique» ne font pas l'histoire d'une science, et un texte ne saurait donc valoir par sa seule importance historique. Il existe en effet au moins une autre manière de considérer le Cours de linguistique générale que celles qu'oppose Christian Puech dans «Pour une histoire de la linguistique dans l'histoire de la linguistique?» (2006) -comme un texte ayant «effectivement joué un rôle séminal dans la genèse des différents structuralismes comme si le texte possédait en lui-même et de manière virtuelle son historicité, le principe de son devenir» (Puech, 2006: p. 18) ou comme une «matrice projective et productive, assez puissante pour ordonner une série de projets scientifiques apparentés (la linguistique des Cercles, le structuralisme "généralisé", les sémiologies, etc.) apparentés, mais aussi assez fondamentalement concurrents et dispersés» (Puech, 2006: p. 19)- et qui ont pour point commun de reposer sur une identification entre histoire des sciences et histoire des idées ${ }^{8} l^{\prime}$ inscrire dans l'histoire du saussurisme, c'est-à-dire le lire à la lumière

5 Voir aussi, notamment, Normand (1995): p. 221-222.

6 Voir également Trabant (2005): p. 118, Normand (1995): p. 221, ainsi que Puech (2000): p. 88/Puech (2008): p. 1098-1099, et Puech (2006): p. 18-19.

7 Rastier a ainsi beau jeu d'ironiser sur la notion de «coupure saussurienne» (voir Rastier, 2015: p. 15 et 51), lui qui fait de la lecture de Saussure «une propédeutique aux sciences du texte» (Rastier, 2015: p. 50) au lieu de se mettre à l'école de ce dernier, pour reprendre une injonction de Bachelard.

8 Le bref commentaire de la distinction bachelardienne entre histoire des sciences périmée et histoire des sciences sanctionnée que l'on trouve dans le même texte témoigne nettement de cette confusion. Voir Puech (2006): p. 12. Les connaissances sanctionnées, en effet, n'échappent en rien à l'historicité, pas plus que les connaissances périmées ne sont reléguées à un «pré-scientifique» dont la démarcation d'avec le scientifique dépendrait de critères de scientificité «posés, en fait, au départ» (Puech, 2006: p. 12). Comme l'a montré Georges Canguilhem (voir Canguilhem, 1968: p. 9-23 et 173-186), cette distinction est inséparable du concept d'obstacle épistémologique, qui implique précisément que toute vérité est nécessairement historique, étant toujours erreur rectifiée. Il s'agit bien, en revanche, d'une «histoire jugée» (Canguilhem, 1968: p. 183), mais parce qu'elle est celle de la pensée, et non des idées. Comme le souligne, de nouveau, Canguilhem, «des valeurs rationnelles doivent ordonner l'histoire de la science puisqu'elles polarisent l'activité scientifique elle-même» (Canguilhem, 1968: p. 183). 
de la théorie saussurienne de la langue, telle qu'elle se donne à lire dans l'ensemble du corpus saussurien'. Je voudrais ainsi pour ma part montrer que le Cours de linguistique générale est le résultat d'une lecture de Saussure, et s'inscrit comme tel dans une histoire qu'il inaugure, mais qui le dépasse, et dont la caractéristique principale est ce que l'on appelle en psychanalyse le principe de répétition. J'envisagerai pour ce faire deux des quatre «réceptions de Saussure» que distingue Christian Puech dans «L'émergence de la notion de "discours" en France et les destins du saussurisme» $(2005)^{10}$-à savoir celles des comptes rendus, des Cercles, du structuralisme généralisé, et des travaux philologiques inaugurés par l'ouvrage de Godel (1957) et l'édition critique du Cours de linguistique générale par Engler (1967/1974)-, substituant à l'analyse de la première l'étude de celle qu'a constitué, avant elle, la rédaction du Cours de linguistique générale, et laissant de côté la troisième, pour m'en tenir au champ de la linguistique proprement dite. Je dérogerai par ailleurs à l'ordre chronologique, en commençant -après une rapide présentation de la théorie saussurienne elle-même-, par la lecture structuraliste de Saussure, afin de mieux mettre en perspective la lecture des éditeurs du Cours de linguistique générale ainsi que celle des néosaussuriens.

\section{La rupture saussurienne}

Il n'est plus de mode de parler de «coupure» ou de «rupture» ${ }^{11}$ saussuriennes, notions que d'aucuns relèguent à une période révolue de l'histoire intellectuelle française, marquée par une «emprise massive du politique» (Normand, 1995: p. 230) ${ }^{12}$, et que d'autres, plus généralement, récusent comme inappropriées à la linguistique qui, comme «science humaine», ne serait pas justiciable d'un modèle discontinuiste ${ }^{13}$. Il me semble pour ma part, tout d'abord, que cette notion de «sciencehumaine», en tantquedistincte de celle de «science de la nature», n'a pour le moment d'autre consistance que son évidence, et qu'elle mériterait au moins d'être élaborée. Il n'y a par ailleurs aucune raison que la pensée scientifique, dans le domaine des sciences dites «humaines», s'élabore selon des lois différentes de celles qui prévalent dans les sciences dites «de la nature», c'est-à-dire que la vérité puisse s'y constituer autrement que comme une erreur rectifiée. En deuxième lieu, il est possible de poser le problème autrement: on peut, au lieu de s'interroger sur l'épistémologie convenant à la linguistique, s'attacher à la constitution et à la définition de l'objet de cette science. Or, force est alors de constater une triple rupture opérée par la théorie saussurienne: avec le mot -ou toute entité linguistique-comme objet donné, et avec la représentation de la langue comme entité en évolution et comme entité délimitée.

Saussure rompt tout d'abord avec l'évidence du donné idiomologique, de l'entité linguistique (mot, forme, syntagme, phrase, etc.) qui s'offre à l'analyse du linguiste. Il dénie toute existence à cette entité linguistique, que l'on considère traditionnellementcommeunobjetanalysable-et à analyser-de différents points de vue: phonique, morphologique, syntaxique, sémantique, mais qu'il construit pour sa part comme résultat, c'est-à-dire comme effet de langue. C'est là, en effet, le sens de la distinction, dans «De l'essence double du langage» $(1891)^{14}$, puis dans les «Notes pour un livre sur la linguistique générale 1 et 2» (1893-1894) ${ }^{15}$, de différents points de vue irréductibles, constitutifs d'objets distincts, et de l'affirmation de la nécessité, en linguistique, de

9 Ce à quoi appelle d'ailleurs également Christian Puech dans d'autres textes. Voir Puech (2000): p. 86-87/ Puech (2008): p. 1098.

10 Voir Puech (2005 : p. 95-97. Voir aussi Puech (2004): p. 125-127.

11 Le concept de rupture épistémologique est un concept de Bachelard, corrélatif de celui d'obstacle épistémologique; Althusser parlait pour sa part plus volontiers de coupure.

12 Voir par exemple Normand (1995), dont la conclusion est cependant ambivalente, mais également Rastier (2015) : p. 15 et 51 (voir la note 7 ci-dessus).

13 Voir par exemple Puech (2006): p. 12-15, ainsi que Chiss \& Puech (1997) : p. 9-10, ou Sériot (1999): p. 28-29 et 310-311.

14 Saussure (2002): p. 23-25.

15 Saussure (2002): p. 197-202. 
«substitu[er] [...] la discussion des points de vue à celle des "faits" » (Saussure, 2002: p. 24). Citons par exemple, extrait du deuxième ensemble de feuillets:

Voici notre profession de foi en matière linguistique: En d'autres domaines on peut parler des choses «à tel ou tel point de vue», certain qu'on est de retrouver un terrain ferme dans l'objet même. En linguistique, nous nions en principe, qu'il y ait des objets donnés, qu'il y ait des choses qui continuent d'exister quand on passe d'un ordre d'idées à un autre, et qu'on puisse se permettre de considérer des «choses» dans plusieurs ordres, comme si elles étaient données par elles-mêmes. (Saussure, 2002: p. 201).

S'il faut parler, ici, de " rupture », c'est dans la mesure où cette affirmation de Saussure était -demeure encore-inouïe: ce donné que l'on avait jusqu'ici pris pour point de départ, pour objet de l'analyse linguistique, Saussure en affirme l'inexistence, c'est-à-dire qu'il le problématise, et assigne à la linguistique pour première tâche de constituer ce donné comme linguistique, d'en définir la linguisticité. Il ne s'agit pas d'analyser l'entité linguistique «du point de vue phonique», «du point de vue de la forme», «du point de vue du sens», «du point de vue synchronique», «du point de vue diachronique», etc., mais de prendre conscience du fait que l'objet, en linguistique, est relatif à, c'est-à-dire constitué par, ces points de vue, et que ce sont donc ces derniers qui définissent l'objet, objet qu'il faut ainsi construire, au sens bachelardien.

Dans ce cadre, Saussure insiste sur deux types d'irréductibilité, sur lesquelles il ne transigera jamais, que ce soit dans ses notes autographes ou dans ses cours de linguistique générale tels que nous les donnent à lire les notes de ses étudiants, entre phonologie et linguistique, d'une part, et entre synchronie et diachronie, d'autre part. La première irréductibilité est corrélative de la mise au jour d'une dualité constitutive du signe, en lieu et place de la dualité traditionnelle, purement empirique, et fondée sur ce donné de l'entité linguistique que Saussure, pour sa part, problématise. On lit ainsi, par exemple, dans «De l'essence double du langage»:

Le dualisme profond qui partage le langage ne réside pas dans le dualisme du son et de l'idée, du phénomène vocal et du phénomène mental; c'est là la façon facile et pernicieuse de le concevoir. Ce dualisme réside dans la dualité du phénomène vocal COMME TEL, et du phénomène vocal COMME SIGNE - du fait physique, (objectif) et du fait physico-mental (subjectif), nullement du fait «physique» du son par opposition au fait «mental» de la signification. Il y a un premier domaine, intérieur, psychique, où existe le signe autant que la signification, l'un indissolublement lié à l'autre; il y en a un second, extérieur, où n'existe plus que le «signe»; mais à cet instant le signe réduit à une succession d'ondes sonores ne mérite pour nous que le nom de figure vocale. (Saussure, 2002, p. 20-21).

Quant à la deuxième, que l'on ne cesse de vouloir ramener à une distinction purement méthodologique, mais que Saussure présente sans ambiguilté comme une distinction in $\mathrm{re}^{16}$, elle introduit pour sa part une deuxième rupture épistémologique (corrélative de la première): avec la représentation de la langue comme entité en évolution. Ce point apparaît de la manière la plus nette dans «Status et motus» (?1894-1897). Saussure affirme en effet dans ce texte:

Il vaut mieux préciser d'emblée Nous ne considérons pas la linguistique comme une

16 J'utilise ici à dessein une expression de Tullio De Mauro, abondamment citée à l'appui d'une conception purement méthodologique de la distinction saussurienne: «On a cru communément que la distinction se place, pour Saussure, in re: l'objet "langue" a une synchronie et a une diachronie, comme monsieur Durand a un chapeau et une paire de gants» (Saussure, 1972 : p. 452, note 176). Il me semble pour ma part que la langue saussurienne a une synchronie et une diachronie comme M. Durand a un chapeau et une paire de gants, mais dans la seule mesure où M. Durand est (tout à la fois, contradictoirement et irréductiblement) son chapeau et sa paire de gants. Là réside précisément la différence entre langue et idiome. Voir ci-dessous. 
science dans laquelle il y a un bon principe de division à ehereher trouver, mais - à part une ou deux réserves comme une science qui essaie d'assembler en un seul tout deux objets complètement disparates depuis le principe, en se persuadant qu'ils forment un seul objet (Saussure, 2002: p. 226).

Or, la langue y est également définie comme un objet synchronique, le point de vue synchronique étant seul susceptible de rendre compte de la propriété de signification inhérente au langage, et dont la théorisation linguistique doit rendre raison: «[...] il se trouve que dans la langue c'est aux états, et à ceux-ci seuls, qu'appartient le pouvoir de signifier; d'autre part la langue hors de ce pouvoir signifier cesserait d'être quoi que ce soit» (Saussure, 2002: p. 226). À l'entité en évolution de la linguistique du XIX ${ }^{\mathrm{e}}$ siècle, plus ou moins organiciste, mais en tout cas historiciste, se substitue ainsi chez Saussure un objet double, synchronique et diachronique. Cette représentation est en rupture radicale avec la représentation spontanée du linguiste, qui conçoit la langue sur le modèle de l'idiome, idiome qui paraît pour sa part exister dans le temps, mais dont Saussure montre qu'il n'a en réalité, comme langue, d'autre existence que synchronique.

La troisième rupture inaugurée par la linguistique saussurienne, avec la représentation de la langue en termes d'entité délimitée qu'accrédite par exemple l'existence de langues nationales ou des dénominations comme «latin», «français», «vieux-haut-allemand», «allemand», apparaît de manière tout particulièrement nette dans le troisième cours ${ }^{17}$ (1910-1911), mais est lisible dès les conférences de 1891, où Saussure reprend à son compte certaines propositions de Gaston Paris et des dialectologues, et affirme que les idiomes ne sont délimités ni dans le temps ni dans l'espace: «[...] il n'y a que des états de langue qui sont perpétuellement la transition entre l'état de la veille et celui du lendemain» (Saussure, 2002: p. 165) et, par ailleurs, "[d]e même qu'il n'y a pas de dialectes délimités, de même il n'y a pas de langues délimitées dans les conditions normales» (Saussure, 2002: p. 172).

On peut résumer cette triple rupture opérée par la théorie saussurienne en parlant de distinction entre langue et idiome: entre les langues (les idiomes) et le langage, termes d'une opposition plurimillénaire, Saussure introduit un troisième terme, la langue, objet spécifiquement linguistique, dont la théorisation permet de construire les idiomes comme un résultat, c'est-à-dire de rendre compte du donné empirique, manifestations du langage que Saussure a été le premier -et, comme nous le verrons, le seul- à problématiser. Saussure définit la langue comme un système de valeurs purement oppositives, relatives, négatives, c'està-dire comme un fonctionnement dont son et sens, en tant que linguistiques, sont les effets. Dans «De l'essence double du langage», le constat de l'absence en linguistique de tout objet donné trouve en effet également à se formuler en termes de négativité, et de différences inséparables d'une combinaison, celle même qui est constitutive du signe: si, écrit Saussure, «[d]ans d'autres domaines [...] on peut parler des différents objets envisagés sinon comme de choses existantes elle[s]-mêmes du moins comme de choses qui résument choses ou [?] entités positives» (Saussure, 2002: p. 65), «il semble que la science du langage soit placée à part : en ce que les objets qu'elle a devant elle n'ont jamais de réalité en soi, ou à part des autres objets à considérer; n'ont absolument aucun substratum à leur existence hors de leur différence ou en LES différences de toute espèce que l'esprit trouve moyen d'attacher à $L A$ différence fondamentale ${ }^{18}$ : mais sans que l'on sorte nulle part de cette donnée fondamentalement et à tout jamais négative, de la DIFFERENCE de deux termes, et non des propriétés d'un terme» (Saussure, 2002:

17 Dans les chapitres consacrés à la diversité géographique (Saussure \& Constantin, 2005: p. 101-143). Voir Toutain (2014): p. 77-98.

18 Figure ici dans le manuscrit, en face en marge, entouré, le segment : «...mais - que leur différence réciproque fait toute leur existence à chacun». 
p. 65); par ailleurs, «non seulement il n'y aura pas de termes positifs mais des différences; mais 2o ces différences résultent d'une combinaison de la forme et du sens perçu ${ }^{19}{ }{ }$ (Saussure, 2002: p. 66). C'est, comme Saussure l'affirme ensuite notamment dans le deuxième cours (1907), en cette délimitation-combinaison -le point de vue synchronique- que consiste la langue:

[...] le rôle <caractéristique $>$ du langage vis-àvis de la pensée ce n'est pas < d'être> un moyen phonique, matériel mais c'est de créer un milieu intermédiaire de telle $<$ nature $>$ que le compromis entre la pensée et le son aboutit d'une façon inévitable à des unités < particulières.> La pensée de sa nature chaotique est forcée de se préciser parce qu'elle <est> décomposée, elle est répartie par le langage en des unités. [...] c'est le fait <en quelque sorte $>$ mystérieux que la pensée-son implique des divisions qui sont les unités finales de la linguistique. [...]

Le terrain de la linguistique est le terrain commun <qu'on pourrait appeler dans un sens très large le terrain $>$ des articulations, c'est-à-dire des «articuli», des petits membres dans lesquels la pensée prend conscience <(valeur? B.)> par un son. (Saussure, 1997: p. 21-22).

Celle-ciest dèslors, non unobjet, fût-ilstructural ou systématique, mais un fonctionnement, dont les idiomes, entités apparemment «positives», tangibles, sont la manifestation. On lit ainsi notamment dans le troisième cours: «Ce fait $<$ linguistique > [la délimitation-combinaison, AGT] donnera naissance à des valeurs qui elles < pour la première fois $>$ seront déterminées, mais qui n'en resteront pas moins des valeurs, avec le sens qu'on peut attacher à ce mot». (Saussure \& Constantin, 2005: p. 285), puis:

Donc on peut envisager tout le système de la langue comme des différences de sons se combinant avec des différences d'idées.
Il n'y a point d'idées positives données, et il n'y a point de signes acoustiques déterminés hors de l'idée. Grâce à ce que les différences se conditionnent les unes les autres, nous aurons quelque chose pouvant ressembler à des termes positifs par la mise en regard de telle différence de l'idée avec telle différence du signe. On pourra alors parler de l'opposition des termes et ne pas maintenir qu'il n'y a que des différences <à cause de cet élément positif de la combinaison> (Saussure \& Constantin, 2005: p. 288-289).

Définie comme fonctionnement, la langue saussurienne n'implique dans sa définition aucune délimitation objectale, que ce soit dans l'espace ou dans le temps. Cette délimitation ne devient nécessaire que lorsqu'on forme le dessein d'étudier un idiome, dans son évolution ou comme état de langue. Par ailleurs, l'extériorité de la langue, qui constitue les idiomes comme tels, ne saurait être qu'une extériorité non objectale. C'est là le deuxième pan de la théorisation saussurienne de la langue, qui consiste en une réélaboration de la notion traditionnelle -héritée notamment de Whitneyde convention, réélaboration dont le cadre est notamment celui de la distinction entre langue et parole qui, significativement, émerge plus tardivement dans l'élaboration saussurienne, à l'époque des cours de linguistique générale, qui en donnent à lire la constitution progressive ${ }^{20}$. Saussure définit la langue comme «le produit social dont l'existence permet à l'individu l'exercice de la faculté du langage» (Saussure \& Constantin, 2005: p. 214), «la partie sociale du langage» (Saussure \& Constantin, 2005: p. 217). Il délimite ainsi la langue dans le tout du langage, en même temps qu'il rend raison du caractère social de la parole, dont la langue constitue tout à la fois un cadre de théorisation et un objet distinct: la parole est ensemble le fonctionnement exécutif de la langue -l'exercice de la faculté de langage, dont la langue constitue la linguisticité, c'est-à-dire que la langue constitue comme linguistique- et, comme y insiste Saussure à

19 «du sens perçu» est une conjecture des Écrits de linguistique générale; le manuscrit est illisible, si ce n'est, peut-être, «sens»; Amacker transcrit: «du sens par n<otre> ( ?)» (Saussure, 2011: p. 109).

20 Voir Toutain (2014): p. 122-150. 
quelque reprise, un objet hétérogène, impliquant notamment la phonologie, et le locuteur. Il en définit par ailleurs le mode d'existence, c'est-àdire l'extériorité, extériorité constitutive, au lieu d'objectale, celle d'un «lien social» (Saussure \& Constantin, 2005: p. 217), d'un «produit historique» (Saussure, 2002: p. 209), toujours hérité, transmis, et n'existant que dans cette transmission, ce qu'il appelle dans le deuxième cours la «vie sémiologique», et qu'il s'efforce longuement de cerner dans les notes sur les légendes germaniques.

C'est donc, à ce qu'il me semble, en termes de distinction entre langue et idiome, que l'on peut caractériser au mieux l'apport saussurien, qui consiste en une théorisation du rapport son/sens constitutif du langage, à quoi renvoie sa définition de la langue comme un système de valeurs. Cette distinction fut cependant recouverte par le structuralisme, qui, après la réception académique des comptes rendus, constitue le premier moment de la réception scientifique du Cours de linguistique générale, entériné comme tel par le développement ultérieur de la linguistique, et ainsi décisif pour le destin de l'œuvre de Saussure.

\section{La réception structuraliste}

Saussure est demeuré, pour l'histoire de la linguistique, le «père du structuralisme». C'est ainsi, en effet, que l'ont lu les structuralistes européens, et par exemple Hjelmslev, Jakobson, Martinet et Benveniste. Il importe peu, à cet égard, que tous se soient par ailleurs montrés critiques -à différents degrés- envers la pensée saussurienne. Mettre en ouvre, en effet -et c'est bien ce queles structuralistes européens ont voulu faire, soit qu'ils s'inscrivent directement dans la lignée de Saussure, soit que, comme Jakobson dans nombre de textes, et pour une part Martinet, ils fassent de Saussure un simple précurseur du structuralisme- ne saurait bien entendu signifier «répéter», ou «accepter intégralement», mais implique au contraire de travailler -au sens du «travail du concept» de Canguilhem ${ }^{21}$-, et, dans ce cadre, potentiellement, d'altérer.

Or, comme je me suis efforcée de le montrer à quelques reprises, du système à la structure ${ }^{22}$, il y a un changement radical de problématique. Si la problématique saussurienne, eu égard à la problématisation du donné et à la construction des idiomes comme effets de langue qui la spécifient, peut être qualifiée d'étiologique, en regard, la démarche structuraliste doit être définie comme «analytique» ${ }^{23}$, puisqu'elle consiste en une analyse (structurale, fonctionnelle, formelle) du donné, au lieu d'une problématisation de ce dernier. La phonologie structuraliste est à cet égard exemplaire ${ }^{24}$. Il s'agit en effet, pour les phonologues, d'intégrer le son à la langue, en procédant à une analyse fonctionnelle de cet aspect du langage, permettant de distinguer entre phonétique et phonologie, ou, plus souplement, entre divers degrés de linguisticité pour établir une hiérarchie fonctionnelle des faits phoniques. Le rapport son/sens, loin d'être théorisé, fait donc figure de principe d'analyse du donné, qui demeure pour sa part non interrogé. Corrélativement, la langue ne fait l'objet d'aucune définition, mais constitue au contraire le cadre de l'analyse. Plus exactement, sa définition commune comme instrument de communication n'est pas remise en cause. Cette double acceptation du donné, sous la forme de l'entité linguistique (de la parole, dans l'acception structuraliste de ce terme) et sous celle de la définition usuelle du langage ou de la langue -celle de la connaissance commune, avec laquelle Saussure permettait pour sa part de rompre-apparaît de manière très nette dans «La théorie saussurienne en rétrospection» (1942), lorsque Jakobson commente le début du

21 Voir Canguilhem (1968): p. 206.

22 Comme le souligne Benveniste dans «"Structure" en linguistique» (1962; voir Benveniste, 1966: p. 92), Saussure n'a en effet jamais employé le terme de structure au sens de «système».

23 Les guillemets signalent que je donne à ce terme - ici et dans l'ensemble de cet article - un sens particulier, et qui - malheureusement: je suis consciente des ambiguïtés que cette homonymie peut générer, et d'autant plus que ce terme est déjà polysémique - m’est propre.

24 Voir Toutain (2015). 
premier paragraphe du troisième chapitre de l'introduction du Cours de linguistique générale, intitulé «La langue; sa définition». Cet alinéa a pour source les «Notes pour un livre sur la linguistique générale 1 et 2» évoquées et citées plus haut, et on y lit ainsi notamment que "c'est le point de vue qui crée l'objet» (Saussure, 1972: p. 23). Jakobson le cite in extenso et l'introduit en ces termes:

La notion de langage est évidente et par conséquent la notion de la linguistique comme science du langage. Cependant Saussure, en posant la question "Quel est l'objet à la fois intégral et concret de la linguistique?», annonce dès le début que "cette question est particulièrement difficile» et finit même par déclarer que l'objet intégral de la linguistique «nous apparait comme un amas confus de choses hétéroclites sans lien entre elles» (24). Pour éclaircir cette conclusion paradoxale du grand linguiste, nous devons suivre de plus près son raisonnement. (Jakobson, 1988: p. 399).

Les citations produites ici par Jakobson sont à replacer dans les développements du troisième cours où Saussure introduit la distinction entre langue et parole ${ }^{25}$ et délimite ainsi la langue dans le tout du langage -qui se caractérise pour sa part par son hétérogénéité, à laquelle il doit d'être inclassable et inappréhendable-, distinction sur la nature de laquelle, comme tous les structuralistes, Jakobson se méprend. Le fait remarquable me paraît néanmoins être la proposition initiale: «La notion de langage est évidente et par conséquent la notion de la linguistique comme science du langage», où apparaît de la manière la plus explicite l'acceptation du donné. À cette définition préalable du langage répondra l'acceptation de cet autre donné qu'est l'entité linguistique. Le passage du Cours de linguistique générale cité ensuite par Jakobson fait ainsi l'objet du commentaire suivant:

Posons-nous à présent la question: est-ce vrai que le langage se distingue par son caractère multiple des autres outils de la civilisation humaine? Essayons d'appliquer mutatis mutandis le même point de vue à un autobus. En imitant le passage cité, on pourrait donc dire qu'un observateur superficiel serait tenté d'y voir un objet technique concret; mais un examen plus attentif y fera trouver successivement trois ou quatre choses parfaitement différentes, selon la manière dont on le considère: comme métal, comme moyen de transport, comme correspondant de l'ancien omnibus à chevaux. Pourtant nous savons bien que ce qui importe avant tout dans tout outil, aussi bien dans la machine que dans le langage, c'est sa fonction. Le procédé rapporté par Saussure, l'examen soi-disant attentif d'un phénomène linguistique reflète exactement non pas ce phénomène comme tel, mais la manière néogrammairienne de l'aborder. C'est l'esprit atomisant de la fin $\mathrm{du}$ siècle qui autorisait les linguistes à traiter le côté phonique, les sons du langage, sans se soucier de leur rôle dans le langage, sans considérer leur fonction linguistique. [...] Par conséquent le point de vue primaire et essentiel est, en dépit de l'opinion citée, objectivement donné. La linguistique envisage tous les éléments constitutifs d'un autobus par rapport aux besoins du transport. (Jakobson, 1988: p. 399-400).

De nouveau, la question sur laquelle s'ouvre ce passage est remarquable puisqu'elle présuppose la définition du langage: il est question des «autres outils de la civilisation humaine», classe dans laquelle le langage s'inscrit donc d'emblée. La comparaison du langage à un autobus est pour sa part tout particulièrement propre à faire apparaître la différence de perspective avec Saussure: il ne s'agit pas, comme chez Saussure, de points de vue constitutifs de l'objet -un autobus est un autobus, et c'est par ailleurs un produit, dont l'existence objectale ne saurait donc être remise en cause, mais est au contraire le point de départ de l'analyse-, mais de points de vue appliqués à un objet. Apparaît ensuite la notion de fonction, qui est explicitement présentée comme un principe d'analyse: il s'agit de «traiter le côté phonique, les sons du langage», en "considér[ant] leur fonction linguistique». Quant aux «éléments 
constitutifs d'un autobus», ils sont bien ce donné idiomologique que Saussure s'efforce pour sa part de constituer comme linguistique. Jakobson fait en revanche de la langue (de la fonction), un principe d'analyse du donné. La langue, corrélativement, est présupposée, au lieu d'être définie.

La définition de la langue comme instrument de communication est également centrale chez Martinet, dont elle fonde la linguistique, linguistique qui est éminemment analytique, comme en témoigne le principe de pertinence, à cet égard emblématique. C'est au nom de ce principe que Martinet refuse la distinction entre langue et parole, à laquelle il substitue la notion de réalité fonctionnelle. Il affirme ainsi par exemple dans «La pertinence» (1973):

Mais la distance qu'il faut nécessairement prendre, au nom de la pertinence, vis-à-vis de l'opposition langue-parole, doit finalement aboutir à son rejet pur et simple. Il est clair que si les traits pertinents de la substance phonique doivent être considérés comme des éléments constitutifs d'une langue -et c'est là le message fondamental de la phonologie-, comme on ne saurait nier qu'ils appartiennent à la parole au sens le plus normal du terme, cela veut dire qu'ils participent conjointement de la langue et de la parole, ce qui rend impossible le maintien de l'opposition. [...]

Ce qu'il ne faut pas hésiter à dire, c'est que la langue est représentée à tous les points $\mathrm{du}$ circuit de la parole. La langue n'est pas mentale ou non mentale. Elle est l'ensemble des comportements, physiques ou psychiques, directement observables ou non, relatifs à un certain type de communication, communs à tous les membres d'une communauté. Elle n'est pas un complexe de «représentations» et "d'images acoustiques», toutes choses qui existent surtout dans la conscience du linguiste qui cherche, par introspection, à se représenter les phénomènes et qui peuvent, sans doute, mais exceptionnellement, accompagner l'exercice de la communication linguistique. Pour étudier la langue, en elle-même et pour elle-même, selon la formule même du Cours, il faut se refuser d'y voir une réalité strictement psychique, ce qui aboutirait à faire, de la linguistique, un chapitre de la psychologie. Il convient de la saisir dans sa réalité fonctionnelle, celle que nous révèle l'application, aux énoncés étudiés, du principe de pertinence. (Martinet, 2000: p. 100).

Il substitue ainsi à la distinction saussurienne entre deux objets de théorisation, corrélative de la constitution d'un concept: la langue, distincte du langage et des idiomes et cadre de théorisation de la parole, une distinction purement analytique entre deux types de réalités d'avance considérées comme linguistiques, la langue et la parole, dont la première est un objet à édifier sur le modèle de l'idiome qui en constitue le support de représentation et dans le cadre de l'analyse de la seconde, et qu'il s'agit dès lors seulement de situer l'une par rapport à l'autre: réalité "purement psychique», atteinte par l'introspection, par opposition à une parole «physique», ou «réalité fonctionnelle» extraite de la parole à laquelle elle est immanente.

L'élaboration structuraliste se fonde donc sur un double donné: celui d'une définition préalable de la langue comme instrument de communication -l'acceptation du donné du rapport son/sens-, et celui de l'entité linguistique, qu'il s'agit seulement de construire, au sens objectal, et non bachelardien, du terme, au moyen d'une analyse linguistique, c'est-à-dire fonctionnelle ou structurale. Aussi n'y a-t-il rien de commun entre le système saussurien et la structure des structuralistes: tandis que Saussure définit la langue comme système, les structuralistes mettent en œuvre ce que l'on peut appeler une «hypothèse structurale», fondée, pour sa part, sur la notion commune de structure -structure constatable en tout idiome, et dont il faudrait ainsi au contraire rendre raison. De même qu'ils reprennent à leur compte- mutatis mutandis selon les auteurs ${ }^{26}$-la définition de la langue comme instrument de communication, les

26 Cette définition n'est pas au cœur de la linguistique hjelmslevienne qui, cependant, ne la remet pas en cause, et dans laquelle elle demeure latente. La critique benvenistienne de cette définition est quant à elle extrêmement ambivalente. Voir Toutain (2016). 
structuralistes se fondent sur une appréhension ou une définition de la langue comme structure pour procéder à une analyse structurale du donné et ainsi construire la langue- c'est-à-dire l'idiome - comme structure: forme (Hjelmslev), instrument de communication (Jakobson et Martinet, de manière diversement élaborée) ou structure informée de signification, « sémiotique» doublée d'une «sémantique» (Benveniste). Cette démarche est tout particulièrement nette dans les textes de Hjelmslev, par exemple dans «Linguistique structurale» (1948), qui s'ouvre sur la proposition suivante:

On comprend par linguistique structurale un ensemble de recherches reposant sur une hypothèse selon laquelle il est scientifiquement légitime de décrire le langage comme étant essentiellement une entité autonome de dépendances internes, ou, en un mot, une structure. (Hjelmslev, 1971: p. 29).

On lit un peu plus loin:

[...] l'hypothèse demande de considérer cette entité autonome comme étant constituée essentiellement de dépendances internes. Elle soutient que l'analyse de cette entité permet de dégager constamment des parties qui se conditionnent réciproquement, et dont chacune dépend de certaines autres et ne serait concevable ni définissable sans ces autres parties. Elle ramène son objet à un réseau de dépendances, en considérant les faits linguistiques comme étant en raison l'un de l'autre. (Hjelmslev, 1971: p. 31-32).

Citonségalement ces affirmations deBenveniste dans «Tendances récentes en linguistique générale» (1954), où le concept saussurien de valeur apparaît comme un principe d'analyse:

Partant de l'expression linguistique native, on procède par voie d'analyse à une décomposition stricte de chaque énoncé en ses éléments, puis par analyses successives à une décomposition de chaque élément en unités toujours plus simples. Cette opération aura pour but de dégager les unités distinctives de la langue, et il y a déjà ici un changement radical de la méthode. Alors qu'autrefois l'objectivité consistait dans l'acceptation intégrale du donné, ce qui entraînait à la fois l'admission de la norme graphique pour les langues écrites et l'enregistrement minutieux de tous les détails articulatoires pour les textes oraux, on s'attache aujourd'hui à identifier les éléments en tant qu'ils sont distinctifs à tous les niveaux de l'analyse. Pour les reconnaître, ce qui n'est en aucun cas une tâche aisée, on s'éclaire de ce principe qu'il n'y a dans une langue que des différences, que la langue met en œuvre un ensemble de procédés discriminatoires. On ne dégage que les traits pourvus de valeur significative en écartant, après les avoir spécifiés, ceux qui ne représentent que des variantes. Une grande simplification est opérée désormais, et il devient alors possible de reconnaître l'organisation interne et les lois d'agencement de ces traits formels. (Benveniste, 1966: p. 8).

Les structuralistes ont ainsi procédé à une lecture de Saussure qu'il faut qualifier $\mathrm{d}^{\prime}$ «idiomologique», dans la mesure où ils se sont référés à la théorie saussurienne -qu'ils se sont efforcés de mettre en œuvre, ou dont ils se sont efforcés de poursuivre l'élaborationsans se départir de la représentation en termes d'idiome avec laquelle l'effort de Saussure avait précisément consisté à rompre, et où ils ont ainsi fait de la langue un principe d'analyse du donné, et de construction des idiomes, c'est-à-dire d'une idiomologie qui, à la lumière récurrente de la théorisation saussurienne de la langue, nous apparaît indûment faite linguistique. Comme nous allons le voir dans ce qui suit, c'est cet obstacle épistémologique de l'idiome qui unifie le saussurisme et scelle, cent ans après la parution du Cours de linguistique générale, la nécessité d'un (nouveau) «retour à Saussure».

\section{Bally et Sechehaye lecteurs de Saussure}

Comme le souligne Christian Puech ${ }^{27}$, «plus le "vrai visage" de Saussure se dessine, et plus les choix de Bally et Sechehaye apparaissent pour

27 Voir la note 9 ci-dessus. 
ce qu'ils sont: des choix dont les motivations doivent être interrogées de manière plus sophistiquée que ce que l'on fait en général à partir de l'alternative trahison/fidélité ou d'autres considérations plus psychologiques qu'historiques» (Puech, 2000: p. 86/2008: p. 1098). C'est, comme le rappelle Estanislao Sofia dans l'introduction de son édition de la Collation Sechehaye ${ }^{28}$, à ce genre d'analyse qu'invitait déjà Rudolf Engler en 1975 dans «European Structuralism: Saussure» lorsqu'il écrivait: «The comparison of Sechehaye's systematic and theoretic views on Saussure with the more intuitive and practical insights of Bally would constitute a fascinating chapter in the history of Saussurian comprehension». (Engler, 1975: p. 833, note 5). Dans cet esprit, mais d'un point de vue un peu différent -résolument épistémologique, et corrélatif d'une lecture nouvelle de Saussure-, mon analyse portera quant à elle sur la lecture de Saussure par Bally et Sechehaye, que je m'efforcerai tout d'abord de caractériser brièvement, en contrepoint d'une lecture du Cours de linguistique générale que cette caractérisation permettra de mettre en perspective $^{29}$. Je procéderai pour ce faire à une rapide analyse de deux échantillons de cette lecture de Saussure, tous deux publiés en 1940: «L'arbitraire du signe. Valeur et signification», de Bally, et "Les trois linguistiques saussuriennes», de Sechehaye.

\section{1. «L'arbitraire du signe. Valeur et signification»}

L'article de Bally est d'autant plus intéressant pour mon propos qu'il fut écrit en réponse aux articles de Pichon et de Benveniste ${ }^{30}$ qui ont ouvert ce que l'on a pu appeler la querelle de l'arbitraire du signe, en soumettant le principe saussurien à la critique. Bally entend en effet faire œuvre saussurienne, en réfutant les arguments avancés par les deux linguistes, selon lesquels «F. de Saussure introduit des termes contradictoires dans la définition du signe» (Bally, 1940: p. 193), et «le lien qui unit le signifiant au signifié, loin d'être arbitraire, a au contraire un caractère de nécessité organique» (Bally, 1940: p. 193), et en «développ[an], à cette occasion, quelques principes fondamentaux touchant la nature $\mathrm{du}$ signe linguistique» (Bally, 1940: p. 193). Il est d'autant plus remarquable, à cet égard, qu'il introduise pour ce faire une distinction entre valeur et signification qui, malgré le contexte, et sa présentation comme telle ${ }^{31}$, n'a rien de saussurien, mais s'inscrirait plus volontiers dans la conception structuraliste, à laquelle il se réfère d'ailleurs également.

Comme il apparaît de la manière la plus nette dansletroisièmecours, ladistinctionsaussurienne entre valeur et signification se présente comme une distinction entre une notion de première venue (la signification) et sa théorisation (dans le cadre du concept de valeur). On lit ainsi au début du chapitre "Valeur des termes et sens des mots»: «C'est peut-être une des opérations les plus délicates à faire en linguistique, de voir comment le sens dépend et cependant reste distinct de la valeur. Là éclate différence entre vue du linguiste et vue bornée considérant la langue comme une nomenclature». (Saussure \& Constantin, 2005: p. 282), avant une définition de la signification comme «contrepartie de l'image auditive» (Saussure \& Constantin, 2005: p. 282), représentation que Saussure s'attache ensuite à modifier en introduisant le concept de valeur, ce quilui permet de conclure, à la fin du chapitre, que le schéma du signe dont il était parti «n'est [...] pas initial dans la langue» (Saussure \& Constantin, 2005: p. 287), «n'est qu'un produit secondaire de la valeur» (Saussure \& Constantin, 2005: p. 286). Bally définit pour sa part la signification comme

28 Voir Sofia (2015): p. XL.

29 Comme le rappelle également Estanislao Sofia (voir Sofia, 2015: p. XL), Engler s'était pour sa part attaché, en 2004, à montrer que Bally et Sechehaye se sont en général abstenus de laisser leurs propres élaborations théoriques infléchir la rédaction du Cours de linguistique générale. Voir Engler (2004).

30 Voir Pichon (1937). L'article de Benveniste est «Nature du signe linguistique», repris dans les Problèmes de linguistique générale, 1 (voir Benveniste, 1966: p. 49-55).

31 Voir Bally (1940): p. 199. 
le «reflet linguistique» (Bally, 1940: p. 194) de la «représentation sensorielle actuelle» (Bally, 1940: p. 194), tandis que le «concept virtuel attaché au mot dans la mémoire, sans aucun contact avec la réalité» (Bally, 1940: p. 194) est la valeur. La seconde relève de la langue, tandis que la première relève de la parole ${ }^{32}$. Il s'agit ainsi pour lui de deux types d'objets linguistiques, qui se partagent ce qu'on entend généralement par sens, là où la distinction saussurienne implique pour sa part la dualité de la langue et de l'idiome (à laquelle se substitue chez Bally, comme chez les structuralistes, une dualité analytique entre langue et parole). Les développements qui suivent sur le «champ associatif» ${ }^{33}$ témoignent ainsi de la positivité de la valeur ballyenne. Apparaît ensuite la métaphore du moule rejetée par Saussure $^{34}$ au profit de la représentation en termes de fonctionnement, et qui montre bien la consistance objectale de la langue ballyenne:

On comprend dès lors sans peine à quel point la valeur doit influer sur la pensée, quand il s'agit des idées abstraites; leur expression, en tant qu'elle est fixée par la langue, oblige l'esprit à louvoyer dans les méandres du vocabulaire pour se couler finalement dans des moules préétablis; dans un cas donné, faut-il dire indolence ou nonchalance, adresse ou dextérité, facile ou aisé, falloir ou devoir? Quiconque a le sens de la propriété des termes trouve le choix sinon facile, du moins naturel; en réalité, il nous astreint à procéder par définitions de mots et non de choses. (Bally, 1940: p. 198-199).

On mesure, à la lecture de ce passage, la radicale nouveauté de la définition saussurienne de la parole comme fonctionnement exécutif de la langue: parler, pour Saussure, ce n'est pas utiliser les mots de la langue -conception commune de la langue comme un instrument de communication, que l'on retrouve ici, mutatis mutandis, chez Bally-, mais «penser langagièrement», «articuler» sa pensée en mots qui se trouvent ainsi -c'est le fonctionnement même de la langue- délimités à nouveau ${ }^{35}$. À ce fonctionnement, Bally substitue la valeur comme lieu du linguistique, de manière dès lors très comparable, paradoxalement, à Benveniste et, comme il apparaît ensuite, aux phonologues, dont il convoque un peu plus loin la théorie à l'appui de son argumentation, ce qui, d'une part, montre qu'il ne perçoit pas la différence de problématique séparant Saussure $\mathrm{du}$ structuralisme, et d'autre part, témoigne de ce que, leur donnât-il les noms saussuriens de «signifiant» et de «signifié», son et sens sont pour lui des donnés, qu'il s'agit seulement de soumettre, chacun pour leur part, à une analyse linguistique, ce qui, comme nous l'avons vu, est la démarche structuraliste:

Ajoutons encore que la même opposition entre valeur et signification régit aussi les formes matérielles de la langue ; là encore, F. de Saussure a vu tout l'essentiel. On lit dans le Cours (p. 150 s.) qu'une même expression Messieurs! prononcée à plusieurs reprises dans une conférence reste identique à elle-même malgré les différences de débit, d'intonation, etc.: qu'est-ce à dire, sinon qu'un signifiant est fixé par la langue (c'est sa valeur), et que les variations qu'il subit dans ses réalisations de la parole constituent, dans chaque cas, sa signification.

Il en est de même des phonèmes isolés; comme le fait remarquer M. Henri Frei, la phonologie de Trubetzkoy opère avec des valeurs phonologiques; mais on sait que l'articulation des phonèmes comporte un certain jeu dans la parole; Saussure dit (p. 164) qu'en français $r$ peut se prononcer grasseyé ou roulé, sans qu'il cesse d'être identique à lui-même (c'est-à-dire sans que sa valeur soit compromise par ces modalités contingentes, qui relèvent de la parole). Dépassant le cadre de la linguistique,

32 Voir Bally (1940): p. 194.

33 Voir Bally (1940) : p. 195-197.

34 Voir Saussure (1997): p. 21 et Saussure (1972): p. 155.

35 Voir en particulier, à cet égard, les développements de «De l'essence double du langage» relatifs à la synonymie, en particulier Saussure (2002): p. 78-79. 
il note encore (même page), que l'identité (autrement dit la valeur) des signes de l'écriture subsiste en regard des infinies fluctuations qu'ils subissent dans leur réalisation. Il remarque enfin (p. 151) qu' on peut reconstruire une rue de fond en comble sans qu'elle cesse d'être la même «parce que l'entité qu'elle constitue n'est pas purement matérielle; elle est fondée sur certaines conditions auxquelles sa matière occasionnelle est étrangère». N'est-ce pas dire que la «valeur» de la rue est distincte de sa «signification»? On entrevoit ainsi l'existence possible d'une science générale des valeurs parallèle à une science générale des significations. (Bally, 1940: p. 201).

Bally cite ici, outre le paragraphe «La valeur linguistique considérée dans son aspect matériel», le chapitre «Identités, réalités, valeurs», sur lequel je reviendrai ci-dessous. Il apparaît cependant dès à présent que Bally en fait -de même que du paragraphe «La valeur linguistique considérée dans son aspect matériel»- une lecture non saussurienne, en conférant à la valeur une consistance objectale. De là les prolongements d'ordre sémiotique qu'il entrevoit, et qui font là encore penser au structuralisme.

\section{2. «Les trois linguistiques saussuriennes»}

Dans «Langue et parole» $(1942)^{36}$, Hjelmslev se réfère, pour caractériser sa propre démarche d'analyse de la distinction saussurienne entre langue et parole, aux propos liminaires de Sechehaye dans «Les trois linguistiques saussuriennes»:

De toutes façons, la vraie critique du Cours consiste à collaborer avec son auteur, soit pour creuser plus avant qu'il n'a pu le faire les assises de la science linguistique, soit pour édifier d'une façon plus définitive la construction dont le Cours n'a pu fournir qu'une première et imparfaite ébauche. (Sechehaye, 1940: p. 3).
Ce texte de Sechehaye nous livre ainsi une lecture de Saussure, et la meilleure qui soit: une lecturecritique, visant à poursuivre la théorisation linguistique. La lecture sechehayenne de Saussure est cependant tout aussi idiomologique que celle de Bally (et des structuralistes), constat qui, de nouveau, est d'autant plus digne d'intérêt que Sechehaye a voulu faire œuvre saussurienne. Ce caractère idiomologique est lisible dès la page suivante, où Sechehaye cite comme exemple de savants s'étant appliqués à «cherche[r] à l'intérieur de la doctrine saussurienne une meilleure application de ses propres thèses» (Sechehaye, 1940: p. 4) l'école phonologique de Prague. Selon Sechehaye, «[t]out le chapitre de la "Phonologie" dans le Cours de Linguistique générale repose sur une conception ambiguë des choses que Saussure n'avait pas eu le temps de tirer au clair» (Sechehaye, 1940: p. 4) et «[1]es linguistes de Prague sont venus débrouiller cet écheveau» (Sechehaye, 1940):

De cette science de la physiologie des sons, que Saussure appelait «phonologie» et que nous appelons couramment «phonétique», ils ont séparé la phonologie proprement dite, la grammaire des phonèmes, et ils ont ainsi constitué une discipline de linguistique statique dont ils ont trouvé d'ailleurs tous les principes dans les pages maîtresses du Cours lui-même. (Sechehaye, 1940: p. 4-5).

Cette «grammaire des phonèmes» était envisagée par Sechehaye lui-même dans son ouvrage de 1908, Programme et méthodes de la linguistique théorique, comme le souligne Jakobson à quelques reprises; il n'en demeure pas moins significatif que Sechehaye ne perçoive pas la différence de problématique qui sépare Saussure des phonologues: dans la radicale exclusion saussurienne de la phonologie, corrélative d'une opposition entre son et son-idée qui rompt avec l'opposition traditionnelle entre son et idée, Sechehaye entend les prémices d'une distinction entre forme et substance, dont le point de départ est précisément le donné du son et du sens, ainsi que de leur rapport.

36 Voir Hjelmslev (1971): p. 79. 
La suite de l'article témoigne de la présence, en arrière-plan, de la représentation idiomologique de la langue comme entité. On lit en effet au début de la section intitulée «Le problème des rapports de la diachronie et de la synchronie», à propos de la distinction entre synchronie et diachronie:

[...] la thèse saussurienne prise non pas à la lettre, mais interprétée à la lumière des idées qui régnaient à l'époque où elle a été formulée, ne comport[e] pas tous les paradoxes qu'on a voulu y voir. Elle revient à établir le fait bien simple et en lui-même évident que l'axe du temps est le lieu où se produisent les événements, en eux-mêmes étrangers au système grammatical, mais qui, agissant sur la langue au travers de la parole amènent des perturbations dans ce système et l'obligent à des réajustements ; en un mot, que l'opposition du diachronique et du synchronique, telle que Saussure l'a vue, revient à celle qu'il y a entre les facteurs contingentiels, qui agissent du dehors sur la langue, et les facteurs organiques, qui soutiennent la langue du dedans. (Sechehaye, 1940: p. 5-6).

Sechehaye précise alors:

[...] nous avons usé évidemment de beaucoup de liberté à l'égard du texte du maître, mais nous croyons, en procédant à une révision de ses principes, avoir continué et précisé sa pensée retenue et déformée par certaines préoccupations qui dominaient la linguistique de l'époque. (Sechehaye, 1940: p. 6).

Il renonce en réalité à la distinction saussurienne dans ce qu'elle a de novateur - car la distinction entre système et histoire, ou entre état et histoire, est comme le souligne Sechehaye lui-même, un «fait bien simple et en lui-même évident», ce pour quoi il est aisé, comme l'a fait Jakobson, suivi par tant d'autres, de lui trouver des «précurseurs». Cette distinction saussurienne entre synchronie et diachronie est en effet corrélative du concept de valeur - une entité purement négative ne saurait avoir d'existence dans le temps - et, comme nous l'avons vu, consiste à substituer à la représentation spontanée - car c'est ainsi que s'offrent les idiomes à notre analyse - de la langue comme une entité en évolution la reconnaissance de deux objets radicalement distincts et irréductibles. Or, la distinction de Sechehaye s'ancre en revanche dans une telle représentation, puisque celuici distingue pour sa part entre deux types de facteurs, respectivement extérieurs et internes, ce qui suppose une entité permettant d'effectuer le partage, et parle d'événements qui « agissent sur la langue au travers de la parole» et «amènent des perturbations dans le système» qui devra ainsi opérer des «réajustements».

Cette représentation sous-tend également la lecture sechehayenne de la distinction langue/ parole, en termes traditionnels d'instrument de communication et d'utilisation de cet instrument: la langue, selon Sechehaye, est «l'ensemble et le système des signes arbitraires en usage à un moment donné dans une société donnée» (Sechehaye, 1940: p. 7), et la parole «l'acte particulier et concret d'un sujet usant de la langue soit pour se faire comprendre, soit pour comprendre» (Sechehaye, 1940: p. 7). C'est elle, enfin, qui est derrière la conception du système comme abstraction idéalisante qui apparaît dans la section «La linguistique synchronique ou des états de langue», lorsque Sechehaye affirme qu'eu égard à «la complexité réelle des faits» (Sechehaye, 1940: p. 12), «[1]a tâche propre de la linguistique statique n'est donc pas d'embrasser tous les faits, mais de dégager de l'ensemble de ces faits ce qui répond dans quelque mesure à l'idéal abstrait d'un état de langue» (Sechehaye, 1940: p. 13). Il faut insister sur le caractère éminemment analytique de cette conception, qui résout sur le plan de l'objet, empiriquement, un problème que Saussure envisage pour sa part théoriquement, en définissant la langue comme un fonctionnement, indépendant, comme tel, de toute délimitation dans l'espace et dans le temps. Sechehaye parlait un peu plus haut d'«image simplifiée, approximative et idéale» (Sechehaye, 1940: p. 11) de l'objet, et le terme de simplifiée donne à penser qu'il pourrait s'agir d'un écho de la proposition du Cours de linguistique générale selon laquelle «la notion d'état de langue ne peut être qu'approximative» (Saussure, 1972: p. 143), et «[e]n linguistique statique, comme dans la plupart des sciences, aucune démonstration n'est possible sans une simplification conventionnelle des données» (Saussure, 1972: p. 143), qui provient de ce passage du troisième cours: 
Il y a une part de convention indéniable à accepter en parlant d'un état. Les limites de ce que nous appelons un état seront forcément imprécises. Ces difficultés qu'on pourrait comparer à ceci: un point n'a aucune dimension - une ligne composée de points en a une. Ou: un plan n'a qu'une seule dimension, par conséquent un volume ne devrait pas pouvoir se composer de plans. Ce qui revient à une convention nécessaire. Il y a quelque chose de semblable quand nous parlons d'un état de la langue. Il y a des espaces de temps où la somme des modifications survenues est presque nulle, alors que d'autres espaces de temps moins considérables se trouvent ailleurs le théâtre d'une somme de modifications très importantes.

\section{[...]}

Pour la rigueur il vaudrait mieux définir un état autrement que par l'absence de changements importants, mais nous ne le pouvons pas. Nous faisons comme les mathématiciens avec leurs plans, ils négligent aussi changements infinitésimaux. C'est la part de convention nécessaire; pour la démonstration des choses on est obligé de les simplifier. (Saussure \& Constantin, 2005: p. 276).

Cette proposition est cependant relative à l'état de langue comme objet d'étude -autrement dit, à l'idiome- non à la langue, pour laquelle la question ne se pose pas puisque, par la rupture qu'elle implique avec la représentation de la langue comme entité délimitée, sa définition rend précisément compte de la nécessité de ces approximations idiomologiques. Il est à cet égard extrêmement significatif, à l'échelle de l'histoire du saussurisme, que cette proposition de Saussure ait polarisé l'attention ${ }^{37}$, alors qu'elle n'a de portée véritable que sur le fond de la théorisation saussurienne de la langue, c'est-àdire lorsqu'elle est clairement située au niveau idiomologique.

\subsection{Le Cours de linguistique générale}

On ne saurait faire de Bally et de Sechehaye des structuralistes. Il apparaît cependant, à l'analyse de leur lecture de Saussure, qu'ils n'ont pas pris la mesure de la rupture saussurienne, ce qui, à la lumière récurrente de la théorie saussurienne, les range aux côtés des structuralistes, dont les rapproche leur méconnaissance de la distinction entre langue et idiome, et leur lecture dès lors idiomologique de Saussure. Cette lecture idiomologique transparaît également dans le Cours de linguistique générale, comme il apparaît par exemple à la lecture du troisième chapitre de la deuxième partie, mentionné plus haut, «Identités, réalités, valeurs», où certaines formulations ou certains ajouts des éditeurs témoignent d'une lecture analogue à celle que propose Bally dans «L'arbitraire du signe. Valeur et signification», conférant à la valeur -dans ce chapitre, à l'identité- une consistance objectale. On lit ainsi notamment:

Qu'est-ce qu'une identité synchronique ? Il ne s'agit pas ici de l'identité qui unit la négation pas au latin passum; elle est d'ordre diachronique, -il en sera question ailleurs, p. 249,- mais de celle, non moins intéressante, en vertu de laquelle nous déclarons que deux phrases comme «je ne sais pas» et «ne dites pas cela» contiennent le même élément. Question oiseuse, dira-t-on: il y a identité parce que dans les deux phrases la même tranche de sonorité (pas) est revêtue de la même signification. Mais cette explication est insuffisante, car si la correspondance des tranches phoniques et des concepts prouve l'identité (voir plus haut l'exemple «la force du vent»: «à bout de force»), la réciproque n'est pas vraie: il peut y avoir identité sans cette correspondance. (Saussure, 1972: p. 150).

Or, tandis que les éditeurs affirment ici que $\mathrm{l}^{\prime}$ «explication» par la correspondance entre «tranche de sonorité» et une «même signification» est «insuffisante», le propos des deux développements qui constituent la source de ce passage du Cours de linguistique générale -issus des deuxième et troisième cours $^{38}$ - est significativement différent. Dans le deuxième cours, Saussure affirme qu'il «faut se référer à un lien quelconque» (Saussure, 1997: p. 22),

37 Voir par exemple Chiss (1978), en particulier p. 105, et Toutain (2008): p. 78-81.

38 Voir Saussure (1997): p. 22-23 et Saussure \& Constantin (2005): p. 226-227. 
avant de demander «Quel est-il?» (Saussure, 1997: p. 22) et de comparer l'identité linguistique (synchronique) à «l'identité du train express de 12h50 et de 5h pour Naples» (Saussure, 1997: p. 22) puis à celle d'une rue que l'on aurait rebâtie. On lit ensuite:

Cette question -sur quoi repose l'identitéest la plus grave parce qu'elle revient tout à fait à la question de l'unité. Il n'y a pas identité si certaines conditions tacites ne sont pas acquises d'avance. Le lien de l'identité <linguistique (il peut d'ailleurs y en avoir plusieurs!) $>$ affecte $<$ donc > l'idée même de l'unité. Dans l'exemple de la rue on peut se demander de quel genre est cette unité: on verra qu'elle est purement négative ou oppositive.

Le lien d'identité repose donc sur des éléments qu'il faut rechercher et par lesquels on touchera de très près les unités. Et cette question des identités finit par <être> la même que celle des réalités linguistiques. (Saussure, 1997: p. 22-23).

Dans le troisième cours, il utilise une comparaison analogue à la première qu'il mobilisait dans le deuxième cours: «Nous faisons souvent des identités comme celle-ci: Un train part à 5h25 de Cornavin, -tous les jours- pour nous il est identique». (Saussure \& Constantin, 2005: p. 226), et affirme en conclusion du développement:

Il faut la correspondance parfaite dans la tranche auditive avec la correspondance appréciablement parfaite dans l'idée évoquée. $<$ Tout le mécanisme de la langue roule autour d'identité et de différence> Remarquons seulement ici que poser la question des unités ou celle des identités, c'est la même chose. (Saussure \& Constantin, 2005: p. 227).

L'identité, pour Saussure, est donc une identité de combinaison, et elle est par ailleurs inséparable d'une «différence», ou, dans les termes du deuxième cours, l'unité est «purement négative et oppositive», ce qui est conforme à la définition de la langue comme divisioncombinaison. Le développement du deuxième cours se clôt d'ailleurs sur l'affirmation $\mathrm{qu}^{\prime} \ll i l$ faut se persuader qu'on n'a pas devant soi des êtres concrets » (Saussure, 1997: p. 23), qui nous reconduit aux affirmations relatives à l'absence de tout objet donné en linguistique. Les éditeurs $\mathrm{du}$ Cours de linguistique générale trouvent pour leur part à cette identité une consistance objectale, et la combinaison -qu'ils entendent dans le cadre de la dualité traditionnelle entre son et sens- leur paraît dès lors insuffisante à la constituer; autrement dit, ils entendent ces développements des deuxième et troisième cours de linguistique générale de Saussure dans le cadre de la double problématique des rapports son/sens et forme/substance que mettront plus tard systématiquement en œuvre les structuralistes : l'identité -la valeur de l'article de Bally- n'est autre que l'un des pôles constitutifs de la distinction entre invariant et variations qu'invite à établir la multiplicité des réalisations, en regard de l'existence d'entités linguistiques, entités linguistiques dont l'existence "concrète» n'est pas remise en cause, et dont la définition traditionnelle comme combinaison d'une forme (d'un signifiant) et d'un sens fournit un cadre d'analyse, cependant que l'appréhension de la langue comme forme, que soutient la représentation en termes d'entité -c'est-à-dire le donné de l'idiome, conçu, par exemple, comme instrument de communication- procure le principe de celle-ci. C'est ce qui apparaît de manière très nette dans l'explicitation, par Bally et Sechehaye, des «conditions» et des «éléments à rechercher» dont il est question dans les notes de Riedlinger du deuxième cours:

Pourquoi peut-on reconstruire une rue de fond en comble sans qu'elle cesse d'être la même? Parce que l'entité qu'elle constitue n'est pas purement matérielle; elle est fondée sur certaines conditions auxquelles sa matière occasionnelle est étrangère, par exemple sa situation relativement aux autres; pareillement, ce qui fait l'express, c'est l'heure de son départ, son itinéraire et en général toutes les circonstances qui le distinguent des autres express. Toutes les fois que les mêmes conditions sont réalisées, on obtient les mêmes entités. Et pourtant celles-ci ne sont pas abstraites, puisqu'une rue ou un express ne se conçoivent pas en dehors d'une réalisation matérielle. (Saussure, 1972: p. 151-152). 
De la notion de réalisation des mêmes conditions, au sens de ce qui distingue une entité d'une autre, à la forme hjelmslevienne, il n'y a en effet qu'un pas. Il est remarquable, à l'égard de cette lecture idiomologique, que les éditeurs du Cours de linguistique générale s'arrêtent à la prudente première formulation du deuxième cours concernant le rapport entre identitéet unité: «[1]a question des identités peut se confondre <en partie> avec celle des unités» (Saussure, 1997: p. 22) et «n'est qu'une complication -du reste féconde- de cette question» (Saussure, 1997: p. 22), que corrigeaient pourtant les affirmations, citées ci-dessus, selon lesquelles la question de l'identité «revient tout à fait à la question de l'unité» et «finit par être la même que celle des réalités linguistiques», ainsi que celle sur laquelle se clôt le développement du troisième cours, également citée ci-dessus:

Le mécanisme linguistique roule tout entier sur des identités et des différences, celles-ci n'étant que la contre-partie de celles-là. Le problème des identités se retrouve donc partout; mais d'autre part, il se confond en partie avec celui des entités et des unités, dont il n'est qu'une complication, d'ailleurs féconde. (Saussure, 1972: p. 151).

Identifier les deux questions, c'est en effet définir l'unité comme un résultat, au lieu de la prendre comme un donné: «Tout le mécanisme dela langue roule autour d'identité et de différence», lit-on dans le troisième cours, et c'est là ce qui définit celle-ci comme fonctionnement, ces jugements d'identité que nous faisons en permanence et qui sont, selon une autre expression, heureuse cellelà, des éditeurs, «articulation de la pensée dans la matière phonique».

Jeviensdementionneruneexpressionheureuse de Bally et Sechehaye. Il faut en effet insister sur l'existence, dans le Cours de linguistique générale, de passages remarquablement conformes aux notes des étudiants, fût-ce au prix d'une perte de vigueur ou de simplifications dues à la suppression d'hésitations et surtout, de la combinaison de sources chronologiquement hétérogènes. Au premier rang d'entre eux figure justement le paragraphe au titre duquel se trouve cette expression, qui ouvre le quatrième chapitre de la deuxième partie, consacré à la valeur linguistique ${ }^{39}$, et dont Lacan fit une lecture structuraliste $^{40}$, mais où Manier, en revanche, trouva une définition de la langue sur laquelle se fonder pour élaborer sa théorie de la psychose, développement rigoureusement saussurien, et qui permet comme tel de mesurer l'importance et la fécondité de la rupture saussurienne ${ }^{41}$. Il me semble donc que si le Cours de linguistique générale, reconstitution $^{42}$ de la pensée saussurienne, est, comme il apparaît à travers cette rapide analyse, critiquable, il faudrait néanmoins plutôt s'étonner, eu égard à cette fondamentale mécompréhension de Saussure, que cette pensée y demeure lisible, et louer ses rédacteurs pour le sérieux et la qualité de leur travail ${ }^{43}$. Cela n'empêche certes pas de penser qu'une publication des notes des étudiants eût pu être préférable, comme l'affirmait, par exemple, Paul Regard, au moment où ce travail fut entrepris ${ }^{44}$, ni d'analyser le Cours de linguistique générale pour ce que les travaux des saussuriens, depuis l'ouvrage inaugural de Robert Godel, ont montré qu'il était: une lecture de Saussure. Il serait au contraire, ce me semble, intéressant, du point de vue épistémologique, de relire le Cours de linguistique générale en s'efforçant de repérer les passages dont la rédaction laisse transparaître la mécompréhension ballyenne et sechehayenne de Saussure, afin de mieux cerner ce qui, dans la radicale nouveauté de la pensée saussurienne, fait obstacle à sa compréhension et ainsi la

39 Voir Saussure (1972): p. 155-158.

40 Voir Lacan (1981): p. 135.

41 Voir Manier (1987), Manier (1995) et Manier (2003), ainsi que Toutain (2014): p. 345 sqq.

42 C'est le terme qu'utilisent Bally et Sechehaye dans la préface du Cours de linguistique générale pour caractériser leur travail. Voir Saussure (1972): p. 9.

43 Sérieux dont, comme le souligne Estanislao Sofia dans son introduction, atteste la Collation Sechehaye du troisième cours.

44 Voir tout récemment à ce sujet Sofia (2015). 
nature des obstacles épistémologiques auxquels se heurte la linguistique ${ }^{45}$, et, par ailleurs, de faciliter le travail des lecteurs de ce dernier, dont certains affirment, avec peut-être quelque raison, qu'il demeure la seule publication aisément lisible et accessible au grand public des idées de Saussure, et qu'il faudra de toute façon continuer de lire, en raison de son importance historique, dont une des conséquences est sa présence dans un grand nombre de travaux.

C'est à un autre type de lecture du Cours de linguistique générale que se livrent les tenants du néo-saussurisme, que je voudrais pour cette raison envisager pour terminer ce rapide parcours des différents moments de l'histoire du saussurisme.

\section{La réception philologique: l'exemple du néo-saussurisme}

Dans «Ontologie et épistémologie de la linguistique dans les textes originaux de Ferdinand de Saussure» (2008), Simon Bouquet écrivait qu'«alors que les Écrits de linguistique générale sont aujourd'hui traduits dans 14 langues, très rares sont les linguistes français qui reconnaissent la radicalité de la révolution conceptuelle qui y apparaît » (Bouquet, 2008: p. 2), puis, citant Culioli et Coursil, qui font exception, ajoute:

Ces prises de position témoignent d'un courant de pensée naissant -désigné, depuis peu, par l'étiquette de néo-saussurisme- auquel se rattachent également d'autres linguistes, comme François Rastier et son équipe de recherche, Jean-Paul Bronckart et ses collaborateurs, MarieJosé Béguelin, ou ma propre équipe de recherche à Nanterre. (Bouquet, 2008: p. 2).

Dans Saussure au futur $(2015)^{46}$, François Rastier paraît cependant se distinguer de ce courant:
Sans nous attarder sur les débats paradoxaux qui s'en tiennent au Cours de linguistique générale et les tenants d'un "néo-saussurisme", nous privilégions ici la lecture de De l'essence double du langage, afin de contribuer à la relecture en cours de l'ensemble $\mathrm{du}$ corpus saussurien. (Rastier, 2015: p. 37).

Le terme de néo-saussurisme ne renvoie donc pas à un paradigme unifié. Je l'utilise ici dans un sens très large, pour désigner une problématique consistant à faire de la lecture des manuscrits l'axe d'une redécouverte de Saussure, au sens de la mise au jour d'un "second Saussure» qui serait radicalement différent de celui du Cours de linguistique générale, Cours de linguistique générale qui aurait ainsi induit en erreur des générations de saussuriens. Comme je l'ai indiqué en introduction, cette problématique me paraît trop simple, ce que je m'attacherai à faire apparaître dans ce qui suit en mettant en évidence un fait remarquable: ces lectures de Saussure sont en réalité tout aussi idiomologiques que celles de Bally, de Sechehaye ou des structuralistes. C'est ainsi, par exemple, que Rastier, qui entend «restituer le projet saussurien» (Rastier, 2015 : p. 13), indique à la page suivante qu'il «s'appuier[a] sur des linguistes saussuriens comme Hjelmslev, Coseriu ou La Fauci» (Rastier, 2015: p. 14), s'inscrivant ainsi dans la lecture structuraliste de Saussure, et affirme, par exemple:

Faute de postulat ontologique, le rapport entre signifiant et signifié ne peut plus reposer sur une relation de représentation, ni même sur une simple correspondance, car les faces du signe ne se correspondent pas: il ne repose que sur la relation entre les plans du langage, entre lesquels il n'y a pas coïncidence, ni comme l'a bien vu Hjelmslev, de conformité. (Rastier, 2015, p. 67).

On retrouve ici la référence à Hjelmslev, mais surtout, son et sens, de même que le rapport son/ sens comme définitoire de la langue, y apparaissent

45 C'est d'ailleurs, de même, ce qui me paraît intéressant à repérer dans la lecture structuraliste de Saussure, et c'est à quoi je m'attache dans mes travaux. Je ne suis d'ailleurs pas sûre que j'eusse pu lire Saussure comme je l'ai lu si je n’avais pas bénéficié de cette «erreur féconde» que fut le structuralisme.

46 Voir également Rastier (dir.) (2013): p. 8. 
comme des donnés. De même, si Coursil, dans Valeurs pures. Le paradigme sémiotique de Ferdinand de Saussure, insiste sur la radicale nouveauté de sa lecture de Saussure, affirmant que «[1]e paradigme saussurien a eu l'étrange destin de n'être jamais né» (Coursil, 2015: p. 29) et parlant de «refoulement radical de son programme des valeurs» (Coursil, 2015: p. 29), sa représentation de la langueen termes de structure à plusieurs niveaux ${ }^{47}$ ne se distingue guère de celle des structuralistes. Je m'attacherai cependant ici, pour son caractère exemplaire, à l'article que Simon Bouquet a publié en 1997 dans Émile Benveniste vingt ans après: «Benveniste et la représentation du sens: de l'arbitraire du signe à l'objet extra-linguistique», et où il s'attache à une analyse de la critique benvenistienne du principe saussurien de l'arbitraire du signe, telle qu'elle se donne à lire dans «Nature du signe linguistique».

Simon Bouquet affirme en ouverture de son article que «[s]on propos est d'illustrer comment la critique de Saussure par Benveniste se construit sur fond d'une perspective en trompe-l'œil» (Bouquet, 1997a: p. 107) et que «[c]ette perspective en trompe-l'œil, c'est celle $\mathrm{du}$ Cours de linguistique générale» (Bouquet, 1997a: p. 107). Il entend ainsi «montrer que, de fait, Benveniste reproche à Saussure des arguments qui ne sont pas les siens» (Bouquet, 1997a: p. 108), et «surtout [...] que la critique de Benveniste visant à dépasser Saussure pourrait bien, sur certains points au moins, être ellemême dépassée par la pensée qui apparaît dans les textes originaux». (Bouquet, 1997a: p. 108), autrement dit proposer une «critique, fondée sur le Saussure des textes originaux, de la critique adressée par Benveniste au Saussure du CLG» (Bouquet, 1997a: p. 117). Cette critique s'inscrit cependant dans la double problématique des rapports son/sens et des rapports forme/ substance qui caractérise le structuralisme européen, et qui est celle de Benveniste dans l'article en question. En témoigne la distinction entre «arbitraire du signifiant» et «arbitraire de la valeur» ${ }^{48}$. Le premier, que Bouquet qualifie de conventionnaliste, et assimile à ceux de Platon, Port-Royal ou Whitney, nous reconduit à la conception traditionnelle du signe. Or, l'apport de Saussure, selon Bouquet, ne consiste pas en une redéfinition de cet arbitraire ${ }^{49}$, mais en son articulation à un deuxième arbitraire, l'arbitraire de la valeur qui, pour sa part, concerne séparément le signifiant et le signifié, dont une appréhension formelle ou structurale se substitue ainsi à la définition comme des valeurs, au sens que je me suis efforcée de faire apparaître plus haut:

La seconde propriété d'arbitraire, distincte de la précédente, est l'arbitraire de la valeur. Ce second arbitraireest double:ils'applique de façon distincte à la valeur du signifiant et à la valeur du signifié. Ici, l'arbitraire n'est plus seulement la contingence d'un signifiant par rapport à un signifié, mais une double contingence à l'intérieur du système d'une langue: celle de tout signifiant par rapport aux autres signifiants, celle de tout signifié par rapport aux autres signifiés. Si ce second arbitraire, contrairement au premier, est un aspect original de la pensée saussurienne, l'articulation de ce double arbitraire de la valeur à l'arbitraire du signifiant n'en constitue pas moins un autre aspect original de cette pensée: car la fondation du phénomène linguistique tient, pour Saussure, au fait que deux valeurs arbitraires, la valeur phonologique et la valeur conceptuelle, soient articulées entre elles par le lien arbitraire qui relie le signifiant au signifié. (Bouquet, 1997a: p. 112-113).

La notion de forme apparaît d'ailleurs un peu plus loin dans le texte ${ }^{50}$ :

Par contre, regardant l'arbitraire de la valeur, si arbitraire et nécessité coexistent là aussi,

47 Voir Coursil (2015): p. 77-78.

48 Voir Bouquet (1997a): p. 112-113. Voir aussi Bouquet (1997b): p. 236 et 286-291.

49 Voir Toutain (2014): p. 98-122 et Toutain (2013).

50 On lit en outre dans Introduction à la lecture de Saussure: «Le second degré de l'arbitraire de la langue [soit l'arbitraire de la valeur, AGT] est celui relatif à la "coupure" opérée par un signe dans la substance à laquelle il donne forme». (Bouquet, 1997b: p. 288). 
leur démarcation correspond à deux postulats théoriques bien distincts. En effet, le lien de nécessité (c'est-à-dire de non-arbitraire) est celui qui noue ensemble les différents termes considérés en tant que formes au sein de l'ensemble du système (c'est là, de nouveau, une nécessité de coexistence-et c'est effectivement cette nécessitélà que défend Benveniste), mais, en l'occurrence, le lien d'arbitraire (c'est-à-dire de non-nécessité) n'est pas un lien de forme à forme: au contraire, il articule une forme linguistique -signifiante ou signifiée- à la substance (phonique ou psychologique) dont cette forme se soutient. (Bouquet, 1997a: p. 119-120).

La langue apparaît en effet dans ce passage comme un existant formel faisant face à une substance, avec laquelle elle entretient, comme chez Hjelmslev, un rapport arbitraire. Autrement dit, paradoxalement, Bouquet rejoint ici la position de Benveniste qui, dans cet article, s'attache précisément, de manière éminemment structuraliste, à constituer la forme comme lieu de la nécessité linguistique ${ }^{51}$. Dans son Introduction à la lecture de Saussure (1997), rejoignant cette fois, tout aussi paradoxalement, Bally et Sechehaye, il affirme de même que la phonologie structurale est un prolongement des intuitions saussuriennes.

Le quadrant défini par l'intersection signifiant/ synchronie, celui de la phonologie synchronique, désigne, de façon patente, la place de la science qui prendra ultérieurement le nom de phonologie. [...] La phonologie synchronique apparaît manifestement, on l'a vu, comme un présupposé fondateur de l'épistémologie saussurienne de la grammaire comparée (et de l'épistémologie programmatique de la grammaire comparée qui s'appuie sur cette épistémologie). Elle est aussi, en elle-même, la matière d'une réflexion «riche en observations et en généralisations nouvelles, concrètes et exactes» ${ }^{52}$, comme le note l'un de ses praticiens émérites, Roman Jakobson. C'est bien ce fait qu'ont reconnu les pionniers de la phonologie dès le premier Congrès international des linguistes à La Haye en 1929, en fondant explicitement cette science renouvelée -la fameuse Proposition 22, présentée par Jakobson, Karczewski et Troubetzkoy, peut être considérée comme son acte de baptême- sur les principes saussuriens que sont la distinction entre langue et parole, le caractère systémique et oppositif de la langue, la nature psychique du fait phonologique, ainsi que la thèse, qui va de pair avec les précédentes, de la différenciation entre le niveau de la substance et celui de la forme. (Bouquet, 1997b: p. 258-259).

Il apparaitt ainsi que l'histoire du saussurisme tout entière, depuis la première publication des idées de Saussure sur la linguistique générale jusqu'au récent et contemporain néo-saussurisme, est marquée par la même lecture idiomologique de Saussure. Le Cours de linguistique générale, bien que critiquable, apparaît ainsi comme un maillon -le premier- d'une histoire qui le dépasse: celle du recouvrement de la rupture saussurienne, et il est à cet égard très remarquable qu'il soit finalement le moins opacifiant de tous. Un siècle après le Cours de linguistique générale, on ne peut donc qu'appeler à un «retour à Saussure», à partir de tous les textes disponibles et $\mathrm{du}$ travail philologique auquel s'appliquent de nombreux saussuriens, et qui substitue la réflexion épistémologique à la vénération de l'autographe et à la problématique de l'histoire des idées, dans laquelle le terme d'histoire conserve un sens regrettablement empirique. Que nous enseigne, sur la langue et sur la linguistique, ce singulier destin de l'œuvre saussurienne de linguistique générale? Trabant insiste dans son article «Faut-il défendre Saussure contre ses amateurs? Notes item sur l'étymologie saussurienne» sur le fait que les préoccupations actuelles sont très éloignées de celles de Saussure ${ }^{53}$. Certes. Mais précisément, qu'est-ce à dire? En particulier, est-il si sûr que la question de l'objet de la linguistique soit «complètement résolue» (Trabant, 2005: p. 113)?

51 Voir Toutain (2016): p. 261-279.

52 Jakobson (1973): p. 290. La référence est donnée par Bouquet. Voir Bouquet (1997): p. 259 , note 1.

53 Voir Trabant (2005): p. 113. 
"[S]on objet est "language"» (Trabant, 2005: p. 113) précise Trabant. Peut-être, cependant, estce là une réponse problématique. Car qu'est-ce, en effet, que ce language qui nous aveugle de son évidence?

\section{Références}

Bally, C. (1940). «L'arbitraire du signe. Valeur et signification», in Le Français moderne, VIIIe année, no 3, p. 193-206. Paris: s.n.

Benveniste, É. (1966 [2002]). Problèmes de linguistique générale, I. Paris: Gallimard.

Bouquet, S. (1997a). «Benveniste et la représentation du sens: de l'arbitraire du signe à l'objet extra-linguistique», in Émile Benveniste vingt ans après, numéro spécial de LINX, p. 107-122. Université Paris X-Nanterre: Centre de recherches linguistiques.

Bouquet, S. (1997b). Introduction à la lecture de Saussure. Paris: Payot.

Bouquet, S. (2008). «Ontologie et épistémologie de la linguistique dans les textes originaux de Ferdinand de Saussure», in Texto! [en ligne], vol. XVIII, no 3 [consultée le 09/09/2009]. Disponible sur Internet: http://www.revue-texto.net/index. php?id=1850.

Canguilhem, G. (1968 ${ }^{54}$ [2002]). Études d'histoire et de philosophie des sciences concernant les vivants et la vie. Paris: Librairie philosophique J. Vrin.

Chiss, J.-L. (1978). «Synchronie/diachronie: méthodologie et théorie en linguistique», in Langages, no 49, p. 91-111. Paris: Larousse.

Chiss, J.-L. \& Puech, C. (1997). Fondations de la linguistique. Études d'histoire et d'épistémologie, deuxième édition remaniée. Louvain-la-Neuve: Duculot.
Coursil, J. (2015). Valeurs pures. Le paradigme sémiotique de Ferdinand de Saussure. Limoges: Lambert-Lucas.

Engler, R. (1975). «European Structuralism: Saussure», in Sebeok, T. A. (éd.), Current Trends in Linguistics, vol. XIII, Historiography of Linguistics, 2, p. 829-886. Paris, La Haye: Mouton.

Engler, R. (2004). "The making of the Cours de linguistique générale», in Sanders, C. (éd.), The Cambridge Companion to Saussure, p. 47-58. Cambridge: Cambridge University Press.

Godel, R. (1957 [1969]). Les Sources manuscrites $d u$ Cours de linguistique générale de $F$. de Saussure. Genève: Droz.

Hjelmslev, L. (1971 [1997]). Essais linguistiques. Paris: Les Éditions de Minuit.

Jakobson, R. (1973 [1979]). Essais de linguistique générale, II. Rapports internes et externes du langage. Paris: Les Éditions de Minuit.

Jakobson, R. (1988). Selected Writings, vol. VIII: Completion vol. I: major works 1976-1980. Berlin, New York, Amsterdam: Mouton.

Kyheng, R. (2007). «Principes méthodologiques de constitution et d'exploitation du corpus saussurien», Texto! [en ligne], vol. XII, no 2 [consultée le 09/09/2009]. Disponible sur Internet: http://www.revue-texto.net/ Saussure/Sur_Saussure/Kyheng/Kyheng_ Corpus-saussurien.pdf.

Lacan, J. (1981). Le séminaire, livre III. Les Psychoses. Paris: Seuil.

Manier, A. (1987). "“Ce fait en quelque sorte mystérieux"», in Psychanalystes, Revue du Collège de Psychanalystes, no 22, p. 113129. Paris: Collège de psychanalystes.

54 Une première édition augmentée parut ensuite en 1983, puis une seconde en 1994. 
Manier, A. (1995). Le Jour où l'espace a coupé le temps. Étiologie et clinique de la psychose. Plancoët: La Tempérance.

Manier, A. (2003). «Le psychotique, Saussure et le psychanalyste», in L'Herne Saussure, p. 285-292. Paris: Éditions de l'Herne.

Martinet, A. (2000). Les Introuvables d'André Martinet (La Linguistique, vol. XXXVI, fasc. 1 et 2). Paris: Puf.

Normand, C. (1995). «La coupure saussurienne», in Arrivé, M. \& Normand, C. (dir.), Saussure aujourd'hui, numéro spécial de LINX, p. 219231. Université Paris X-Nanterre: Centre de recherches linguistiques.

Normand, C. (2000). Saussure. Paris: Les Belles Lettres.

Pichon, É. (1937). «La linguistique en France. Problèmes et méthodes», in Journal de psychologie normale et pathologique, XXXIV année, p. 25-48. Paris: Librairie Félix Alcan.

Puech, C. (2000). «L'esprit de Saussure: réception et héritage (l'héritage linguistique saussurien: Paris contre Genève», Modèles linguistiques, tome XXI, fasc. 1, pp. 79-93.

Puech, C. (2004). «Saussure and structuralist linguistics in Europe», in Sanders, C. (éd.), The Cambridge Companion to Saussure, p. 124-138. Cambridge: Cambridge University Press.

Puech, C. (2005). «L'émergence de la notion de "discours" en France et les destins du saussurisme», in Langages, no 159, (Linguistique et poétique du discours. À partir de Saussure, Chiss, J.-L. \& Dessons, G. dir.), p. 93-110. Paris: Larousse.

Puech, C. (2006). «Pour une histoire de la linguistique dans l'histoire de la linguistique?», in Histoire Épistémologie Langage, tome XXVIII, fasc. 1, p. 9-24. Paris: SHESL.
Puech, C. (2008). «Qu'est-ce que faire l'histoire du "récent" ", in Durand, J., Habert, B., Laks, B. (eds), Congrès mondial de linguistique française - CMLF'08, Paris, Institut de linguistique française, pp. 10931102. Disponible sur Internet: http://www. linguistiquefrançaise.org ou http://dx.doi. org/10.1051/cmlf08334.

Rastier, F. (1985). «L'œuvre de Hjelmslev aujourd'hui»; in Caputo, C. \& Galassi, R. (éd.), p. 109-125. Lecce: Il Protagora.

Rastier, F. (dir.) (2013). De l'essence double du langage et le renouveau du saussurisme (Arena romanistica. Journal of Romance Studies, no 12: numéro spécial à l'occasion du centenaire de la mort de Ferdinand de Saussure (1857-1913)). Bergen: University of Bergen, Department of Foreign Languages.

Rastier, F. (2015). Saussure au futur. Paris: Les Belles Lettres.

Sechehaye, A. (1908). Programme et méthodes de la linguistique théorique. Psychologie $\mathrm{du}$ langage. Paris: Honoré Champion/Leipzig: Otto Harrassowitz/Genève: A. Eggimann \& Cie.

Sechehaye, A. (1940). «Les trois linguistiques saussuriennes», in Vox Romanica, no V, p. 1-48. Zurich-Erlenbach: Erigen Rentsch Verlag/Genève: Librairie E. Droz.

Saussure, F. (de) (1972 [1995]). Cours de linguistique générale, édition de Tullio de Mauro. Paris: Payot.

Saussure, F. (de) (1967/1974). Cours de linguistique générale, édition critique par Rudolf Engler. Wiesbaden: Otto Harrassowitz.

Saussure, F. (de) (1986). Le Leggende germaniche. Este: Libreria Editrice Zielo.

Saussure, F. (de) (1996). Premier Cours de linguistique générale (1907), d'après les cahiers d'Albert Riedlinger. Oxford, New York, Séoul, Tokyo: Pergamon. 
Saussure, F. (de) (1997). Deuxième Cours de linguistique générale (1908-1909), d'après les cahiers d'Albert Riedlinger et Charles Patois. Oxford, New York, Tokyo: Pergamon.

Saussure, F. (de) (2002). Écrits de linguistique générale. Paris: Gallimard ${ }^{55}$.

Saussure, F. (de) \& Constantin, É. (2005). «Ferdinand de Saussure: Notes préparatoires pour le cours de linguistique générale 1910-1911, Émile Constantin: Linguistique générale. Cours de $M$. le professeur de Saussure 1910-1911», in Cahiers Ferdinand de Saussure, no 58, p. 83289. Genève: Droz.

Saussure, F. (de). (2011). Science du langage. De la double essence du langage. Édition des Écrits de linguistique générale établie par René Amacker. Genève: Droz.

Seriot, P. (1999). Structure et totalité. Les origines intellectuelles $d u$ structuralisme en Europe centrale et orientale. Paris: Puf.

Sofia, E. (2015). La «Collation Sechehaye» du Cours de linguistique générale de Ferdinand de Saussure, édition, introduction et notes. Leuven, Paris, Bristol, CT: Peeters.
Toutain, A.-G. (2008). «Empirisme, évidence et idéologie. Quelques enjeux $\mathrm{du}$ "dépassement" jakobsonien de l'opposition saussurienne synchronie/diachronie», in Bulletin de la Société de linguistique de Paris, tome CIII, fasc. 1. p. 63-116. Louvain: Peeters.

Toutain, A.-G. (2013). «Dualité et unité de l'arbitraire saussurien», Cahiers Ferdinand de Saussure, no 66, p. 121-135. Genève: Droz.

Toutain, A.-G. (2014). La rupture saussurienne. L'espace $d u$ langage. Louvain-la-Neuve: AcademiaBruylant.

Toutain, A.-G. (2015). La problématique phonologique. Du structuralisme linguistique comme idéologie scientifique. Paris: Classiques Garnier.

Toutain, A.-G. (2016). Entre langues et logos. Une analyse épistémologique de la linguistique benvenistienne. Berlin: De Gruyter.

Trabant, J. (2005). «Faut-il défendre Saussure contre ses amateurs? Notes item sur l'étymologie saussurienne», in Langages, no 159 (Linguistique et poétique du discours. À partir de Saussure, Chiss, J.-L. \& Dessons, G. dir.), p. 111-124. Paris: Larousse.

55 Toutes les citations de ce texte ont été corrigées à la lumière des manuscrits, et les deux leçons peuvent donc différer. Je n'ai pas indiqué les modifications effectuées, pour ne pas alourdir l'appareil de notes. 